\title{
Neuro research presentation sessions at ECR 2020
}

\author{
Philippe Demaerel ${ }^{1}$ (D)
}

Received: 28 January 2020 / Revised: 7 February 2020 / Accepted: 12 February 2020 / Published online: 6 May 2020

(C) European Society of Radiology 2020

\section{Neuroradiology research presented at ECR 2020: main findings, novelties, and remaining questions}

The greatest novelties in the research presentation sessions (RPS) on neuroimaging at the European Congress of Radiology (ECR) 2020 are related to progress in advanced magnetic resonance imaging (MRI) techniques and artificial intelligence (AI). But, as is the case every year at ECR, advances in the fields of stroke, brain tumours, neurodegenerative disease, and multiple sclerosis (MS) are included in the programme too. All abstracts are published in the ECR 2020 Book of Abstracts [1], with session and presentation numbers mentioned in brackets.

In brain tumour imaging, the recently revised WHO classification has resulted in an increasing use of deep learning in the study of new biomarkers. The central focus is the methods in isocitrate dehydrogenase (IDH) genotype prediction and the presence or absence of $\mathrm{O}^{6}$-methylguanine-methyltransferase (MGMT) in glioblastoma. In a preliminary study of 25 patients, classifiers provided natural boundaries for efficient discrimination of IDH-wild type and IDH-mutation (RPS 1011a4). The usefulness of rCBV measurements to differentiate the IDH genotype is also reported (RPS 1011a-7).

The proteomic footprint of IDH-mutation is the presence of 2-hydroxy-glutarate (2HG). The authors studied 39 patients and found $80 \%$ sensitivity and $75 \%$ specificity of single voxel MR spectroscopy in the detection of 2HG (RPS 1011a-5).

The study of the topographical distribution of MGMT promoter methylation in 436 IDH-wild type glioblastoma revealed no statistically significant differences between methylated and unmethylated status (RPS 1011a-6).

Philippe Demaerel

philippe.demaerel@uzleuven.be

1 Department of Radiology, University Hospital KU Leuven, Herestraat 49, 3000 Leuven, Belgium
There is an increasing use of radiomics for volumetry and for assessing response to treatment in brain tumour imaging. In a series of 124 patients, it was shown that it was possible to predict patient survival by analysing radiomics features quantifying tumour intensity and heterogeneity (RPS 1011b-3).

In stroke imaging, several abstracts focus on improvement in stroke patient selection for thrombolysis. One study reports that the mothership and drip-and-ship strategies do not differ significantly in impact on outcome, provided that 'bridging' intravenous thrombolysis is given to the acute stroke patient in the drip-and-ship strategy (RPS 1411b-1).

Automated attenuation measurements on CT angiography source images present excellent performance in detecting ischaemia in acute stroke patients and bear the potential to reduce the use of CT perfusion (RPS 1411b-7). AI can provide an e-CT angiography collateral score for patients eligible for thrombectomy (RPS 1411b-8). Automated assessment of regional hypoattenuations was able to identify expert-classified visual ASPECTS cut-off values and may be helpful for patient selection for thrombectomy (RPS 1411b-9).

Dynamic CT angiography weighting (arterial/arteriovenous/venous) did not impact collateral grade analysis and was not useful in predicting infarct size and clinical outcome (RPS 1111-5).

High-resolution intracranial vessel wall imaging has been used to study the atherosclerotic plaque features and differences were observed in patients with a good and poor leptomeningeal collateral status which may help determine therapeutic decision-making (RPS 1111-6).

Another focus in stroke research deals with postthrombectomy imaging.

The post-thrombectomy CT perfusion parameter time-topeak was an independent predictor of treatment failure and $\mathrm{CT}$ perfusion parameters improve the treatment outcome prediction in acute stroke patients beyond clinical/angiographic parameters, ASPECTS, and pre-treatment perfusion parameters (RPS 1411b-2).

The AURORA meta-analysis provides data on 458 patients who underwent thrombectomy more than 6 hours after last 
known well and showed a considerable long-term clinical benefit in these stroke patients (RPS 1411b-4).

The main reasons of failed endovascular thrombectomy in the posterior circulation are different from those in the anterior circulation: futile vascular access, a failed passage of the occlusion, and interventional complications (1411b-5).

In 400 subjects without prior cardiovascular disease who underwent MRI, serum glucose concentration was identified as a quantitative determinant of white matter lesion volume and the authors suggest that the effect of diabetes on white matter lesion volume might be driven by impaired glucose tolerance (RPS 1111-3).

Neurodegenerative diseases are always high on the list in neuroimaging research. The segmentation and subclassification of white matter hyperintensities were correlated with the cognitive scores on neuropsychological tests and revealed its potential usefulness as biomarker (RPS 611a-1). A novel 3D deep brain model was developed with promising diagnostic accuracy for diagnosis of Alzheimer disease (RPS 611a-4).

Parkinson disease (PD) is also one of the highlights of the research presentation session on neurodegenerative disease (session 611a). With diffusion basis spectrum imaging, it was possible to quantify the dendritic and neuronal loss with oedema and inflammation in the substantia nigra of PD patients (RPS 611a-6). A good correlation was found between the changes of the "swallow tail sign" on quantitative susceptibility mapping and the striatal uptake on PET (RPS 611a-7). With resting-state fMRI, it was possible to show increased connectivity of the left and the right frontoparietal network in PD (RPS 611a-8). After dopaminergic treatment, a decoupling of the right frontoparietal network was observed together with an increase of the ipsilateral connectivity in the left frontoparietal network (RPS 611a-8).

Histogram analysis of diffusion tensor imaging metrics in PD with cognitive decline revealed increased axial diffusivity in the early stages and increased radial diffusivity and mean diffusivity in the later stages in the grey matter. A loss of white matter diffusion restriction is already present in the early stages (RPS 611a-9). The dopamine transporting imagingspecific binding ratio and the mean diffusivity on diffusion tensor imaging can contribute to the discrimination of early PD and rapid eye movement sleep behaviour disorder (RPS 611a-11).

The orientation dispersion index can be generated from the neurite orientation dispersion and density imaging sequence and appears to be a measure of regional neurite changes in early PD (RPS 611a-12).

Measurement of brain atrophy in multiple sclerosis (MS) with a 30-year follow-up suggests that, when present within 5 years of disease onset, it predicts disability a decade or more later (RPS 611b-1). The bi-caudate ratio is an easily performed measurement that can be used as a surrogate marker of global cerebral white mater atrophy in MS. In a meta-analysis, the authors suggested that a trans-sectional cervical cord area of $71 \mathrm{~mm}^{2}$ could be used to discriminate progressive forms of MS from non-progressive forms (RPS 611b-3).

A machine learning-derived model including texture analysis mainly related to deep grey matter was able to predict physical disability in MS patients (RPS 611a-1).

Microscopical fractional anisotropy, obtained from tensorvalued diffusion encoding, is not affected by fibre dispersion and improves the characterisation of white matter pathology in MS (RPS 611b-10).

High correlations were observed between MR planimetric and volumetric measurements and the easily applicable planimetric measurements may therefore give information on brain volume changes which may then be used as a predictor of disability in MS (RPS 611b-11).

Three research presentation sessions (1411a, 1411c, and 1611) are dedicated to advances in functional MRI techniques. One of them is dedicated to arterial spin labelling (ASL), a promising technique that gives an insight in the brain perfusion without the need of gadolinium (Gd) (sessions 1411a). In Moyamoya disease, it is possible to differentiate between affected and unaffected vascular territories (RPS 311-11). ASL has been used successfully in sickle cell vasculopathy (RPS 1411a-2) and porphyria (RPS 1411a-7), in migraine patients (RPS 1411a-10) and in localising the epileptogenic focus (RPS 1411a-11).

One of the most attractive research presentation sessions deals with advanced MRI techniques (1411c). One of these innovative techniques is MRI-guided high-intensity focused ultrasound, a non-invasive treatment for targeted ablation of the ventral intermediate nucleus and the inferior zona incerta. 3D MRI fingerprinting and MRI compilation (MAGIC) are of potential interest because these may result in a considerable reduction of scan time. The technique has been applied for myelin measurements and for diffusion-weighted imaging in stroke patients. Several abstracts deal with resting-state fMRI, tractography, and connectometry.

Inevitably, some attention is paid to gadolinium retention in the brain (session 311). One abstract reports on calvarial Gd deposition and its relationship to hypophosphatemia (RPS 311-2). Other researchers noted the lack of increased signal intensity after more than four gadobutrol administrations (RPS 311-5) or the absence of T1 relaxation time shortening after the serial injection of 11 doses gadobutrol (RPS 311-6).

In summary, fascinating research is presented in the different sessions providing very interesting observations in various research domains of neuroimaging. The major limitation is often the small case series; however, the presentations will undoubtedly generate new ideas and/or foster the research in neuroimaging. 
Funding information The authors state that this work has not received any funding.

\section{Compliance with ethical standards}

Guarantor The scientific guarantor of this publication is Philippe Demaerel.

Conflict of interest The authors of this manuscript declare no relationships with any companies, whose products or services may be related to the subject matter of the article.

Statistics and biometry No complex statistical methods were necessary for this paper.
Informed consent Written informed consent was not required for this study because it summarizes congress proceedings.

Ethical approval Institutional Review Board approval was not required.

Methodology

- Not applicable

\section{Reference}

1. ECR 2020 Book of Abstracts (2020) Insights imaging. https://doi. org/10.1186/s13244-020-00851-0

Publisher's note Springer Nature remains neutral with regard to jurisdictional claims in published maps and institutional affiliations. 